\title{
Analysis and Characterization of Pre-Diffusion in Multi-Linear Silicon Drift Detectors
}

\author{
Andrea Castoldi, Chiara Guazzoni, Member, IEEE, Robert Hartmann, Paolo Madoglio, Student \\ Member, IEEE, and Lothar Strüder
}

\begin{abstract}
Multi-Linear Silicon Drift Detectors (MLSDD) are silicon detectors in which signal electrons are confined within parallel drifting columns at few micrometers from the anodes' surface and transported towards point-like anodes by an electrostatic field. During the initial motion across the thickness the signal electrons spread in all directions to a given width - we call this phenomenon pre-diffusion - until the signal electrons are focused in the drift channels which suppress any further spread into adjacent channels. This work is devoted to the study of the initial motion of the electron cloud when the electrons are being focused toward the minimum of the potential energy. We developed an analytical model to predict the initial charge spread and carried out a detailed measurement campaign to evaluate the impact of the detector operating conditions and of the charge injection level on the initial charge spread. The design of the electron spread during pre-diffusion allows optimization of MLSDD for different applications.
\end{abstract}

Index Terms-Multilinear Silicon Drift Detectors, Electron dynamics, 2D position sensing, imaging detectors

\section{INTRODUCTION}

$\mathrm{S}_{\mathrm{a}}^{\mathrm{n}}$ ILICON Drift Detectors [1] were invented in 1983 by Gatti and Rehak as two dimensional position-sensitive detectors for ionizing particles. Since their invention detector technology and detector architecture have made a substantial evolution. As a consequence silicon drift detectors are nowadays employed as particle tracking detectors in highenergy physics experiments [2-4] and have opened to novel field of application in high-resolution X-ray spectroscopy and imaging with dedicated topologies [5, 6].

Multi-anode Silicon Drift Detectors allows dramatic reduction in the number of channels required for true $2 \mathrm{D}$

Manuscript received August 26, 2008, revised November 17, 2008. This work was supported in part by INFN, Sezione di Milano.

Andrea Castoldi, Chiara Guazzoni and Paolo Madoglio are with Dipartimento di Elettronica e Informazione, Politecnico di Milano and INFN, Sezione di Milano, 20133 Milano, Italy. (telephone +39 02 23996321, e-mail Andrea.Castoldi@polimi.it, telephone: +39 02 23996147, e-mail: Chiara.Guazzoni@mi.infn.it).

Robert Hartmann is with PNSensor GmbH, 80803 München, Germany and with Max Planck Institut Halbleiterlabor, 81739 München, Germany (e-mail roh@hll.mpg.de).

Lothar Strüder is with Max Planck Institut für Extraterrestrische Physik, Giessenbachstrasse, 85741 Garching, Germany and with Max Planck Institut Halbleiterlabor, 81739 München, Germany. $\mathrm{He}$ is also with Universität Siegen, FB Physik, Emmy-Noether Campus, 57068 Siegen, Germany. position sensing and naturally simplify the interconnection issue between detector chip and the front-end electronics. In the case of conventional multi-anode position-sensing silicon drift detectors the final width of the electron distribution in the direction transverse to the drift (i.e. the lateral direction) is an increasing function of the total drift time. The signal charge can be shared by several contiguous anodes, depending on both the operating drift field and the maximum drift path. However if the spread of the signal electrons increases with the drift time, the resolution is suboptimal either for smaller or greater drift distances [7].

A recent evolution, started in the last decade [8], of multianode Silicon Drift Detectors for position sensing are MultiLinear Silicon Drift Detectors [1] (hereafter MLSDDs). They are silicon drift detectors in which signal electrons are confined within parallel drifting columns (or channels) - by means of a proper combination of deep $\mathrm{p}$ and $\mathrm{n}$ implants - at few micrometers from the front surface - within a High Energy n-type implant - and transported towards point-like anodes by means of an electrostatic field with readout times of only few microseconds. Moreover the deposited energy can be measured with spectroscopic resolution at (or very close to) room temperature thanks to point-like anodes of very low capacitance ( $<100 \mathrm{fF}$ ) readout by on-chip JFETs [12].

Signal electrons, generated at a given detector depth (depending on the type of incident particles), are focused by the component of the electric field, directed along the detector depth (hereafter depletion field), toward the detector front surface, until they reach one of the drift channels. While being focused in the drift channel the signal electrons drift toward the anode owing to an almost uniform electric field component (hereafter drift field). Signal holes are immediately collected at the $\mathrm{p}^{+}$strip closest to the interaction point.

At a first level of approximation the electron cloud spread is isotropic during the focusing toward the drift channels. This initial diffusion process (called pre-diffusion), lasts few tens of nanoseconds and stops when the signal electrons reach the drift channels. At low levels of charge injection (when electrostatic repulsion is negligible) the broadening of the charge cloud during pre-diffusion is due to thermal diffusion only. At high levels of charge injection, a further and often dominant broadening contribution arises from electrostatic repulsion. The charge distribution is then "frozen" into the drift channels and electrons are transported to the readout 
anodes without further broadening in the lateral direction, irrespective of the drifted distance, due to the lateral potential barriers generated by the tailored pattern of deep $p$ and $n$ implants. Only longitudinal broadening occurs when electrons move inside the drift channel. Therefore in this transport scheme signal sharing is independent on the drift distance.

The previously highlighted features of MLSDDs make them suitable for 2D particle tracking with position resolution much better than the anode segmentation and independent on the interaction point. Moreover, the suppression of the lateral diffusion improves the capability to handle multi-hit events and the maximum processing rate with respect to conventional multi-anode drift detectors.

A careful design of the drift channel pitch together with an appropriate choice of the biasing/technological detector properties allows tailoring pre-diffusion so that charge division occurs between two-channels for nearly all events opening the way to center-of-gravity interpolation techniques to improve position resolution. On the opposite side minimization of charge sharing between channels is required to enhance the maximum event rate and the peak-background ratio in X-ray spectroscopy imaging applications.

This paper is devoted to the experimental study of the initial motion of the electron cloud in MLSDDs when the electrons are being focused toward the minimum of the potential energy near the deep implanted surface in order to evaluate the impact of the detector properties and operational parameters on the charge cloud lateral spread. Moreover we propose a simplified analytical model to evaluate the impact of thermal diffusion on the charge cloud width. Section II is devoted to the description of the main features of MLSDDs. Section III presents the basic analytical model to evaluate the dependence of the charge cloud lateral width on the detector properties and operational parameters. Section IV deals with the experimental characterization describing the setup and the method used to deconvolute the charge cloud width from the experimental measurements. Section V discusses the obtained results.

\section{MLSDD ARCHITECTURE}

Fig. 1 shows the basic architecture of a MLSDD. An n-type high resistivity silicon bulk (with thickness ranging from about $250 \mu \mathrm{m}$ up to $450 \mu \mathrm{m}$ ) is fully depleted by means of reverse biased $\mathrm{p}$ strips located on both surfaces of the detector. The p-type strips implanted on the back side act as entrance window for the radiation. A high-linearity resistive divider biases the finely structured $\mathrm{p}+$ strips implanted on the front side thus providing the drift field responsible for electron transport toward the collecting anodes. A high energy n-type implant locates the drift channel at few micrometers from the detector front side. A proper combination of deep p-implants (channel-stops) and deep n-implants (channel-guides) confines the generated charge within parallel channels as in fully-depleted pn-Charge Coupled Devices [13]. The on-chip front-end stage (JFET in source follower configuration) buffers the low anode capacitance and provides the output signal at low impedance level to the preamplifier therefore assuring high energy resolution. Deep n-implants account also for drift velocity enhancement [14] as they reduce the amplitude of the residual perturbations of the drift field in the channel induced by the segmentation of the front-side $\mathrm{p}+$ implant in strips.

Fig. 2 shows the simulated electrostatic potential in a plane parallel to the detector surface located at the depth where the potential minimum for the electrons is located. The figure shows the effect of both the drift field, which transports the electrons towards the anodes, and the deep implants which generate lateral barriers between the drift channels.

The MLSDD prototype used for the present work is a large area detector, featuring $240 \mathrm{drift}$ channels, each $120 \mu \mathrm{m}$-wide and $10.2 \mathrm{~mm}$-long. Fig. 3 shows the measured drift time as a function of the drift coordinate with the detector biased at $450 \mathrm{~V} / \mathrm{cm}$ drift field together with the deviations from the linear fit. Proper electron drift is achieved over the full detector length at an average velocity higher than $83 \%$ of the nominal drift velocity (i.e. mobility times the average drift field) which reflects the achieved degree of spatial uniformity of the drift field.

\section{Simplified Model of Charge Cloud Pre-Diffusion}

The electron cloud generated by ionizing radiation spreads owing both to thermal diffusion and to electrostatic repulsion, while being focused towards the drift channels (pre-diffusion). In this section we derive a simplified model that estimates the width of the charge cloud (assuming a point-like ionization occurring close to the back of the wafer at low levels of charge injection) at the moment in which its lateral broadening is frozen by the confining action of the lateral barriers. Once the charge cloud reaches the drifting channel its longitudinal spread (i.e. along the drift coordinate) goes on until it is collected by the anode. At low levels of charge injection (i.e. case of negligible electrostatic repulsion) we suppose that the charge cloud broadens only due to thermal diffusion. Within a first order approximation, electrostatic repulsion is negligible provided that the mean electrostatic energy per electron is lower than the thermal energy $3 / 2 \cdot k_{\mathrm{B}} T$, being $k_{\mathrm{B}}$ the Boltzmann's constant and $T$ the absolute temperature. Although simplified, this approach well approximates the interaction due to few $\mathrm{keV}$ X-rays.

The width of the charge cloud, when focused within the drift channels, depends on the depletion field strength and on the starting distribution of the generated electrons. In fact, the higher the depletion field, the shorter the time needed to focus electrons into the channel (interval during which thermal diffusion takes place) and the smaller the charge cloud width.

In the first instants after generation the electron cloud is spread in an isotropic way by thermal diffusion. The spread along the lateral direction ( $y$-axis) goes on until the electrons are close enough to the deep implanted surface (let us define this depth $z=z_{\mathrm{f}}$ ) so that the lateral potential barriers are high 
enough to prevent the lateral diffusion. Following [9], we assume that the minimum stopping barrier is equal to few (e.g. one, two or three) thermal voltages $V_{t h}=k_{\mathrm{B}} T / q$ (where $q$ is the electron charge). In order to derive $z_{\mathrm{f}}$, we approximate that the amplitude $\Delta V$ of the confining potential barrier decays exponentially with the depth $z$ as it would occur for a pure sinusoidal perturbation (having spatial period $P_{\mathrm{y}}$ ) at the detector surface:

$$
\Delta V(z)=\Delta V_{0} \cdot \exp \left(-2 \pi \cdot \frac{z}{P_{\mathrm{y}}}\right)
$$

where $\Delta V_{0}$ is the amplitude of the first harmonic of Fourier decomposition of the confining potential at the surface. The amplitude of the confining barrier as a function of the depth according to (1) is shown in Fig. 4a in the case of $\Delta V_{0}=1 \mathrm{~V}$ being $P_{\mathrm{y}}=120 \mu \mathrm{m}$ the lateral pitch of channel stops and of channel guides. For example for $\Delta V\left(z_{\mathrm{f}}\right)=2 k_{\mathrm{B}} T / q$, from (1) we get $z_{f, 2 V_{t h}}=55.5 \mu \mathrm{m}$. Fig. $4 \mathrm{~b}$ shows the depth, $z_{f, V_{t h}}, z_{f, 2 V_{t h}}$ and $z_{f, 3 V_{t h}}$ as a function of the amplitude of the first harmonic of Fourier decomposition of the confining potential at the surface.

The rms width of the electron cloud is then given by the well-known expression for thermal diffusion:

$$
\sigma_{\mathrm{f}}=\sqrt{2 D_{\mathrm{n}} t_{\mathrm{f}}}
$$

where $D_{\mathrm{n}}$ is the diffusion coefficient for electrons and $t_{\mathrm{f}}$ is the time required for the depletion field to move the electron cloud to the depth $z_{\text {f. }}$. In this simplified model the focusing time $t_{\mathrm{f}}$ does not depend on the interaction coordinate along the drift direction since we suppose that the charge moves only along the $z$ coordinate until it reaches the channel and starts drifting to the anode. This is not rigorous especially in the cases of depletion field comparable with the drift field. Under normal operating conditions, the depletion field is higher than the drift field (at least a factor of 6), so the previous approximation is acceptable.

The focusing time $t_{\mathrm{f}}$ can be obtained by solving the equation of motion of one electron across the substrate along the $z$-axis:

$\frac{d z}{d t}=-\mu_{\mathrm{n}} \cdot E_{\mathrm{z}}(z)$

where $\mu_{\mathrm{n}}$ is the electron mobility and $E_{\mathrm{z}}$ the depletion electric field. The latter can be computed by solving the Poisson's equation for the electric potential. We assume that the doping concentration of the bulk $\left(N_{\text {sub }}\right)$ is uniform (as it really is) and we approximate the high energy implanted region with a layer of uniform doping $\left(N_{H E}\right)$ and with thickness equal to the full width half maximum of the high-energy implant profile $\left(F W H M_{H E}\right)$ located at the projected range $(P R)$ of the implant itself. Since it results that $z_{\mathrm{f}}$ is located in the detector volume between the high energy implant and the back-side, we are interested in evaluating the electric field only in that region, that is:
$E_{z}(z)=$

$=\frac{q N_{\text {sub }}}{\varepsilon}\left[z+\frac{\varepsilon}{q N_{\text {sub }}} \frac{\Delta V}{d}+\frac{P R}{d} F W H M_{H E}\left(\frac{N_{H E}}{N_{\text {sub }}}-1\right)-\frac{d}{2}\right]$ (4)

where $\varepsilon$ is the silicon dielectric constant, $\Delta V$ is the voltage difference applied between the front and back side, $d$ is the substrate thickness.

The solution of (3), where the electric field is given by (4), with the initial condition $z(0)=d$ is:

$$
\begin{aligned}
z(t)= & e^{-t / \tau} \cdot\left[d+\frac{\varepsilon}{q N_{\text {sub }}} \cdot \frac{\Delta V}{d}+\frac{P R}{d} \cdot F W H M_{\mathrm{HE}} \cdot\left(\frac{N_{\mathrm{HE}}}{N_{\text {sub }}}-1\right)-\frac{d}{2}\right]+ \\
& -\left[\frac{\varepsilon}{q N_{\text {sub }}} \cdot \frac{\Delta V}{d}+\frac{P R}{d} \cdot F W H M_{\mathrm{HE}} \cdot\left(\frac{N_{\mathrm{HE}}}{N_{\text {sub }}}-1\right)-\frac{d}{2}\right]
\end{aligned}
$$

for every $t$ such that $z(t)>P R+F W H M_{\mathrm{HE}} / 2$, where $\tau=\varepsilon / q \mu_{\mathrm{n}} N_{\text {sub }}$ is the characteristic time constant of the motion across the substrate.

The focusing time $t_{\mathrm{f}}$ can be found by imposing $z\left(t_{\mathrm{f}}\right)=z_{\mathrm{f}}$ in (5) and solving for $t_{\mathrm{f}}$ :

$t_{\mathrm{f}}=\tau \log \left[\frac{d+\frac{\varepsilon}{q N_{\text {sub }}} \cdot \frac{\Delta V}{d}+\frac{P R}{d} \cdot F W H M_{\mathrm{HE}} \cdot\left(\frac{N_{\mathrm{HE}}}{N_{\mathrm{sub}}}-1\right)-\frac{d}{2}}{z_{\mathrm{f}}+\frac{\varepsilon}{q N_{\text {sub }}} \cdot \frac{\Delta V}{d}+\frac{P R}{d} \cdot F W H M_{\mathrm{HE}} \cdot\left(\frac{N_{\mathrm{HE}}}{N_{\text {sub }}}-1\right)-\frac{d}{2}}\right]$

The doping profiles and doping levels used for the detector production ensure that $z_{f}>P R+F W H M_{\mathrm{HE}} / 2$ for all considered operating conditions. Equation (6) shows that $t_{\mathrm{f}}$ is directly proportional to $\tau$ and it shows a logarithmic dependence on the thickness of the substrate and on the voltage difference applied between front and back surfaces. Fig. 5 shows the focusing time computed with (6) for two different thicknesses of the substrate (typical values of 300 $\mu \mathrm{m}$ and $450 \mu \mathrm{m}$ have been chosen) and for two different values of substrate resistivity $(5 \mathrm{k} \Omega \cdot \mathrm{cm}$ and $15 \mathrm{k} \Omega \cdot \mathrm{cm})$ as a function of the voltage applied at the back side, when the front side is biased at $-12 \mathrm{~V}$. It results that $\tau$ is of the order of ten nanoseconds. It has to be noticed that for back-side voltages more negative than $-200 \mathrm{~V}$ the two curves relative to a thickness of $300 \mu \mathrm{m}$ show negligible dependence on the substrate resistivity since the considered voltage range is much higher than the depletion voltage. The same consideration is valid in the case of a thickness of $450 \mu \mathrm{m}$ for back-side voltages more negative than $-230 \mathrm{~V}$ when the substrate is over-depleted. For back-side voltages close to the depletion voltage proper of the considered thickness and doping, the focusing time tends to diverge as expected since the depletion field at $z_{f}$ is very low. From (2) we can compute $\sigma_{\mathrm{f}}$ that results of the order of ten micrometers for normal operating conditions (a least 30V of over-depletion).

The main limitations of the proposed model are the followings. First it considers the height of the lateral confining barrier as a Heavyside function so that no diffusion occurs when the barrier is higher than $2 \cdot k_{\mathrm{B}} T / q$. Moreover the barrier amplitude $(1 \mathrm{~V})$ has been roughly estimated by means of 
simulation according to the expected values of the substrate and deep implants doping which have not been directly measured. Let us also remark that this theoretical 1-D model does not take into account the non uniformity of the drift field along the longitudinal and lateral directions, which is present during the pre-diffusion motion causing coupling between the motions along different axes. 3D electrostatic simulation would provide more accurate predictions about the lateral width of the charge cloud.

Finally the model neglects electrostatic repulsion which is not negligible even at low levels of charge injections. If the electron distribution is uniform within a sphere of radius $R$, the mean electrostatic energy per electron is

$$
E_{\text {rep }}=\frac{3}{5} \cdot \frac{q^{2} N}{4 \pi \varepsilon \cdot R}
$$

$N$ being the number of electrons (the factor $3 / 5$ depends on the particular charge distribution considered). It follows from (7) that, if $N=6 \cdot 10^{3}$ (this is the amount of charge injected for the measurements reported in Section V.A) and $R=6 \mu \mathrm{m}$ (equal to the rms width of the laser spot), the mean electrostatic energy per electron is about $3 \cdot k_{\mathrm{B}} T$. Thus electrostatic repulsion starts causing a further broadening of the charge cloud even at such a low level of charge injection.

\section{EXPERIMENTAL CHARACTERIZATION}

\section{A. Setup}

A pulsed semiconductor infrared laser $(\lambda=904 \mathrm{~nm})$ with pulse duration below $1 \mathrm{~ns}$ was used to generate the signal electrons. The laser intensity, calibrated with the lines of a ${ }^{241} \mathrm{Am}$ source, has been adjusted to generate from $6 \cdot 10^{3}$ up to $10^{5}$ electrons. The laser spot has been focused by microscope optics on the back side of the detector. The electrons, generated at some tens of microns from the back surface, spread while moving towards the opposite surface. The voltage applied at the detector back side has been changed in the range $-110 \mathrm{~V}$ (close to the depletion voltage) $\div-200 \mathrm{~V}$ in order to test the impact of the detector biasing condition on the measured lateral spread. The drifting channels sample the electron cloud at the end of the focusing path and the lateral profile can be derived from the charge collected by the readout anodes at the end of the drift. A sampling interval smaller than the anode pitch has been achieved by measuring the charge collected at the same anode and moving the laser spot in the lateral direction ( $y$-axis) by steps of $5 \mu \mathrm{m}$.

The output of the integrated source follower was fed to a voltage preamplifier (gain $=10$ ) during the measurements at low levels of charge injection. The preamplifier output feeds a gaussian shaper, with $250 \mathrm{~ns}$ shaping constant time.

\section{B. Charge Cloud Width Extraction from Experimental Data}

Two methods for estimating the charge cloud width are proposed in [15]. Both of them rely on the fact that the amount of charge collected in a certain drift channel can be written as:

$Q(y)=-q \int_{-L}^{L} n\left(y^{\prime}-y\right) d y^{\prime}$

where $y$ is the lateral displacement of the laser spot with respect to the channel center, $2 L$ is the channel width and $n(y)$ is the charge density at the y coordinate. The integral in (8) can be also thought as the convolution integral between a weighting function $w(y)$ relative to the anode and $n(y)$. In (8) the weighting function is a unit-amplitude rectangle having width equal to $2 L$ (i.e. all the charge between two adjacent lateral barriers cannot escape over them and it is collected in the central channel).

The first method to derive $n(y)$ in [15] takes the first derivative of $Q$ with respect to $y$

$$
\begin{aligned}
& \frac{d Q}{d y} \propto \frac{d}{d y}[n * w](y)= \\
& =\frac{d}{d y} \int_{y}^{+L} n\left(y^{\prime}-y\right) d y^{\prime}-\frac{d}{d y} \int_{y}^{-L} n\left(y^{\prime}-y\right) d y^{\prime}= \\
& =n(-L-y)-n(L-y)
\end{aligned}
$$

The two addends in the last term of (9) do not overlap provided that the width of $n(y)$ is small compared with the channel width $2 L$. Assuming $n(y)$ to be gaussian, the two terms do not overlap if $3 \sigma_{\mathrm{f}}<L$; in our case it is $\sigma_{\mathrm{f}}<20 \mu \mathrm{m}$.

This technique is not suitable at high level of charge injection since as $\sigma_{\mathrm{f}}$ increases, the two addends in the last term of (9) overlap.

The second technique in [15] is more accurate. Assuming

$n(y)=A \cdot \exp \left[-\frac{y^{2}}{2 \sigma_{f}^{2}}\right]$

the integral in (8) becomes:

$Q(y)=-q \sqrt{\frac{\pi}{2}} \cdot A \cdot \sigma_{f} \cdot\left[\operatorname{erf}\left(\frac{y+L}{\sqrt{2} \sigma_{f}}\right)-\operatorname{erf}\left(\frac{y-L}{\sqrt{2} \sigma_{f}}\right)\right]$

$\operatorname{erf}(x)$ being the error function of $x$. Standard fitting technique are then applied to find the charge cloud width $\sigma_{f}$.

The method we propose in this paper is an evolution of the latter. Instead of fitting the experimental data with Eq. (11) we use a deconvolution algorithm to get $n(y)$ from (8). The deconvoluted waveform shows a gaussian shape, as expected. We fit the deconvoluted Gaussian waveform with (10) applying a non-linear least-mean-square algorithm that provides the best estimation of the amplitude $A$, and of the standard deviation $\sigma_{\mathrm{f}}$. Finally the fit results are verified by calculating analytically the convolution of (10) with the rectangular weighting function $w(y)$.

All the experimental data that we have recorded show that there is an offset superposed to the collected charge, while we expect that the charge collected will be zero if the laser spot is focused far away from the observed drift channel. This offset is due to the laser halo, which is much wider than the laser spot. Since this offset does not hold any useful information, it is subtracted from experimental data, before performing the 
deconvolution process. The proposed algorithm provides a good estimation of $\sigma_{\mathrm{f}}$ at low and medium levels of charge injection. Fig. 6 summarizes the results of the steps of the proposed algorithm: in Fig 6(a) the measured output voltage after offset removal is represented together with the weighting function $w(y)$. Fig 6(b) represents the results of the deconvolution and its fitting using a gaussian function as in (10).

\section{EXPERIMENTAL MEASUREMENTS AND DISCUSSION}

\section{A. Low level of charge injection}

The laser intensity has been initially adjusted to generate $6 \cdot 10^{3}$ electrons, in order to study the pre-diffusion of signal electrons at low level of charge injection, i.e. when electrostatic repulsion is supposed negligible respect thermal diffusion. Measurements have been repeated by varying the back side bias voltage (i.e. changing the depletion field intensity) in order to verify (6) and by focusing the laser spot at two different drift coordinates in order to verify that prediffusion does not depend on the drift. All the measurements have been performed with a drift field equal to $450 \mathrm{~V} / \mathrm{cm}$ and applying $-12 \mathrm{~V}$ at the anode region of the front side. The first set of measurement aimed to directly measure the charge focusing time. To this aim we irradiated the detector both on the front-side and on the back side at $500 \mu \mathrm{m}$ drift distance from the collecting anode while keeping the setup identical so that all the electronics delays remain constant. The focusing time has been measured as the time difference between the absolute drift time when irradiating the detector at the same drift coordinate on the front side and the back side. The resolution on such measurement results from the resolution on the individual measurement that is $2 \mathrm{~ns}$. The results are shown in Fig. 7 together with the behavior foreseen by (6) for a $420 \mu \mathrm{m}$ thick $15 \mathrm{k} \Omega \cdot \mathrm{cm}$ wafer.

Fig. 8 shows the measured charge distribution (i.e. amount of charge collected at the anode) as a function of the lateral position of the laser spot in case of back voltage equal to 180 $\mathrm{V}$ and of drift coordinate equal to $500 \mu \mathrm{m}$. The offset due to the laser spot halo has been subtracted from the measured data. The pulse has a flat top since all the generated charge is collected by one anode without charge sharing between adjacent channels, until the laser generates charge within the drift channel. The FWHM of the measured charge distribution is nearly equal to the channel width $(120 \mu \mathrm{m})$ as expected. The shoulders of the charge distribution retain the information related to the rms width of the electron cloud. As expected from (6), the shoulders of the measured charge distribution are sharper as the back side voltage is higher.

We have extracted the charge cloud widths from measurements similar to the one shown in Fig. 8 and repeated as a function of the back side voltage, according to the procedure described in Section IV. Since the laser spot has a finite dimension $\sigma_{\text {laser }}$ (being estimated from independent measurements to be about $6 \mu \mathrm{m}$ ), the contribution of the pre- diffusion has been depurated of the laser spot width, assuming gaussian distribution of the charge cloud density. Fig. 9 shows the obtained results together with the lateral spread derived from the focusing time shown in Fig. 7 according to (2). For normal operating conditions $\left(V_{\text {back }}>150 \mathrm{~V}\right.$ ) the charge cloud width does not depend on the interaction drift coordinate (the difference is less than $1 \%$ ) and is less than $10 \mu \mathrm{m}$ at $200 \mathrm{~V}$ back side voltage. According to (6), an increase of the back voltage produces a reduction of the charge cloud width, which is more pronounced at low back side voltages, due to the logarithmic law in (6). The measured charge cloud width at $110 \mathrm{~V}$ (close to the minimum value assuring the depletion of the detector) can be explained with the fact that the depletion electric field is quite low and the potential minimum for the electrons tends to be located out of the high-energy implanted region. Therefore local statistical variation of the doping concentration can affect significantly the local electric field. Moreover, at such low value of the depletion electric field, the pre-diffusion motion is more significantly coupled with the field component along the drift direction and the lateral direction. This fact is also at the basis of the smaller lateral width computed with the measured focusing time according to (2) that tends to the measured values when the depletion field is much higher than the drift field. Moreover, even if the charge injection level is low, the mean electrostatic energy per electron is comparable to the thermal energy, as computed in Section III. Thus electrostatic repulsion causes a further broadening of the charge cloud that is neglected by (2).

Another interesting point is the study the dependence of the drift time on the lateral coordinate. Fig. 10 shows the drift time as a function of the lateral position of the laser spot in the case of a back voltage equal to $180 \mathrm{~V}$ and of an injected charge of 6,000 electrons. The behavior is identical both for the measurement relative to $500 \mu \mathrm{m}$ drift coordinate and for the one relative to $6740 \mu \mathrm{m}$. The drift time remains constant within the deep $\mathrm{n}$ implanted area. The decrease of the drift time outside the measured channel is due to time walk effects. The interesting point is the $8 \mathrm{~ns}$ drift time increase that occurs when the laser is displaced at lateral coordinates corresponding to the deep-p implanted region. We have explained this time difference with the fact that when the laser injects photons in this region the generated charge will drift for the first time instants in a region where the longitudinal profile of the drift field is affected by larger perturbations than in the deep-n implanted region [14]. Such time difference increases when the back side voltage is raised (for example the drift time increases by $17.5 \mathrm{~ns}$ at $200 \mathrm{~V}$ back voltage) and tends to disappear at low back voltages $(5 \mathrm{~ns}$ at $160 \mathrm{~V}$ back voltage and no appreciable difference for $150 \mathrm{~V}$ or for lower voltages). This is due to the simultaneous occurrence of different phenomena. At higher back voltages, as evident in Fig. 9, the lateral spread of the charge cloud is lower, therefore the electrons actually drift below the deep $n$ implanted region in the middle of the channel when the laser is impinging on the center of the channel (and below the deep 
p implanted region when the laser is impinging on the rim of the channel) so that the wings of the charge distribution do not experience a more rough (smoother) drift field. The second effect is due to the actual trajectory of the charge cloud in reaching the drifting channel. At lower back voltages the time spent by the electrons at depths where the effect of the deep implanted regions is negligible is longer and therefore the time difference caused by the deep implanted regions is not visible anymore.

\section{B. High level of charge injection}

We repeated the measurements described in Section V.A at higher levels of charge injection, in order to evaluate experimentally the additional broadening due to mutual Coulomb interaction. The back side voltages have been changed over a narrower range $(150 \div 200 \mathrm{~V})$, within the normal operating conditions for this prototype of MLSDD.

We measured the charge distribution as a function of the lateral position of the laser spot at different back voltages and for different levels of charge injection. As the injected charge is increased obviously the output amplitude increases, since a larger amount of charge is collected at the anode. Fig. 11 shows a zoom of the measured collected lateral charge profile that is of the charge collected at the anode as a function of the lateral position of the laser spot in the case of a back voltage equal to $200 \mathrm{~V}$ for different levels of charge injection. As shown in Fig. 11 the output pulse becomes wider: the effect of repulsion - dominant in the first nanoseconds after charge generation - further broadens the charge cloud. This effect is evident if we observe the extracted lateral width $\left(\sigma_{\mathrm{f}}\right)$ of the charge cloud - after quadratic subtraction of the laser spot rms width - that is shown in Fig. 12 as a function of the injected charge. Obviously the intensity of the electrostatic repulsion is more significant as the level of charge injection increases and the increase is non linear. For each value of the injected charge, it has been verified that increasing the back voltage reduces the lateral diffusion, as shown in Fig. 13. The increase of the back voltage reduces only the lateral spread due to thermal diffusion. The lateral spread due to Coulomb interaction is nearly insensitive to the back voltage since its contribution is significant only in the first nanoseconds after charge generation, when the electron cloud density is very high. It is interesting to notice that, also for higher level of charge injection, the rms lateral widths extracted from the measurements at $x=6740 \mu \mathrm{m}$ and $x=500 \mu \mathrm{m}$ show a larger difference at lower values of the back side voltage. As already explained the low value of the depletion field (at low back voltages) causes a more pronounced distortion of the electron trajectory close to the collecting anode.

Fig. 14 shows the drift time as a function of the lateral position of the laser spot in the case of a back voltage equal to $200 \mathrm{~V}$ and of an injected charge of 60,000 electrons. The behavior is similar to the one shown in Fig. 10, but not exactly identical. First of all the two measurements relative to $500 \mu \mathrm{m}$ drift coordinate and to $6740 \mu \mathrm{m}$ are qualitatively similar but they do not show a quantitatively identical behavior. The drift time increase that occurs when the laser is displaced at lateral coordinates corresponding to the deep-p implanted region is $51 \mathrm{~ns}$ at $500 \mu \mathrm{m}$ drift coordinate and $64 \mathrm{~ns}$ at $6740 \mu \mathrm{m}$. Moreover the maximum drift time increase occurs for lateral coordinates that are misaligned with respect to the deep implants pattern as visible in Fig. 13. This can be caused by the increase of the charge cloud width due to electrostatic repulsion.

\section{CONCLUSIONS}

In this paper we have presented a detailed experimental study of the initial motion of the electron cloud when the electrons are being focused toward the minimum of the potential energy near the deep implanted surface in order to evaluate the impact of the detector properties and operational parameters on the charge cloud lateral spread. Moreover we have proposed a simplified analytical model to evaluate the impact of thermal diffusion on the charge cloud width that is in reasonable agreement with the experimental measurements. We have directly measured the charge focusing time towards the drifting channel and the impact of the position of charge generation on the drift time.

\section{ACKNOWLEDGMENT}

We acknowledge the staff of the Max Planck Institut Halbleiterlabor in Munich together with H. Soltau and P. Holl, and the staff of PNSensor GmbH in Munich for detector production. We want to acknowledge S. Masci for careful and professional detector bonding. We also thank D. Fugazza for his help in the first phase of the experimental measurements.

\section{REFERENCES}

[1] E.Gatti, P.Rehak, "Semiconductor Drift Chamber: an application of a novel charge transport scheme", Nucl. Instrum. Meth. A225, pp. 608614, 1984.

[2] A. Rashevsky et al., "Characteristics of the ALICE Silicon Drift Detector”, Nucl. Instrum. Meth. A 461, pp. 133-138, 2001.

[3] R.Bellwied et al., STAR SVT Group, "Development of large linear silicon drift detectors for the STAR experiment at RHIC", Nucl. Instrum. Meth., A377, pp. 387-392, 1996.

[4] E. Gatti, P. Rehak, "Review of semiconductor drift detectors", Nucl. Instrum. Meth. A541 pp. 47-60, 2005.

[5] P. Lechner, et al., "Silicon drift detectors for high resolution room temperature X-ray spectroscopy,” Nucl. Instrum. Meth., A 377, pp. 346351, 1996.

[6] P.Lechner, et al., "Silicon drift detectors for high count rate X-ray spectroscopy at room temperature", Nucl. Instrum. Meth., vol. A 458, pp. 281-287, 2001.

[7] E. Crescio, D. Nouais, "Electron cloud size measurement in silicon drift detectors and spatial resolution improvement", Nucl. Instrum. Meth.Vol. 564, No. 1, pp. 475-481, Aug. 2006.

[8] A. Castoldi, P. Rehak, P. Holl, "A New Silicon Drift Detector With Reduced Lateral Diffusion," Nucl. Instrum. Meth., vol. A377, pp. 375380, 1996.

[9] A. Castoldi, P. Rehak, P. Holl, "Signal charge sharing in multilinear drift detectors: design and experimental characterization", IEEE Trans. Nucl. Science, vol. 44, no.2, pp. 134-141, 1997.

[10] A. Castoldi, A. Galimberti, C. Guazzoni, P.Rehak, R.Hartmann, L.Strüder, "Multi-linear silicon drift detectors for X-ray and Compton imaging" Nucl. Instrum. Meth. A, Vol. 568, No. 1 pp. 89-95, 2006. 
[11] A.Castoldi, A.Galimberti, E.Gatti, C.Guazzoni, P.Rehak, L.Strüder, "Xray 2-D Position-Sensing with Multi-Linear Silicon Drift Detectors", IEEE Trans. Nucl. Sci., Vol. 53, No. 2, April 2006, pp. 601-606.

[12] V. Radeka, et al., "Implanted silicon JFET on completely depleted highresistivity devices", IEEE Electron Device Lett. 10 (1989) 91.

[13] L.Strüder, H.Bräuninger, U.Briel, R.Hartmann, G.Hartner, D.Hauff, N.Krause, B.Maier, N.Meidinger, E.Pfeffermann, M.Popp, C.Reppin, R.Richter, D.Stötter, J.Trümper, U.Weber, P.Holl, J.Kemmer, H.Soltau, A.Viehl, C.v. Zanthier, "A $36 \mathrm{~cm}^{2}$ large monolithic pn-charge coupled device X-ray detector for the European XMM satellite mission," Rev. Sci. Instrum., vol. 68, pp. 4271-4274, 1997.

[14] A. Castoldi, C. Guazzoni, L. Strüder, "Effects of Deep n-Implants on the Electrons'Transport in Silicon Drift Detectors", IEEE Trans. Nucl. Sci. Vol. 49, no. 3, pp. 1055-1058, 2002.

[15] R. Bellwied et al., "Studies of dynamics of electron clouds in STAR silicon drift detectors," Nucl. Instumr. Methods, vol. A 439, pp. 507-512, 2000 .

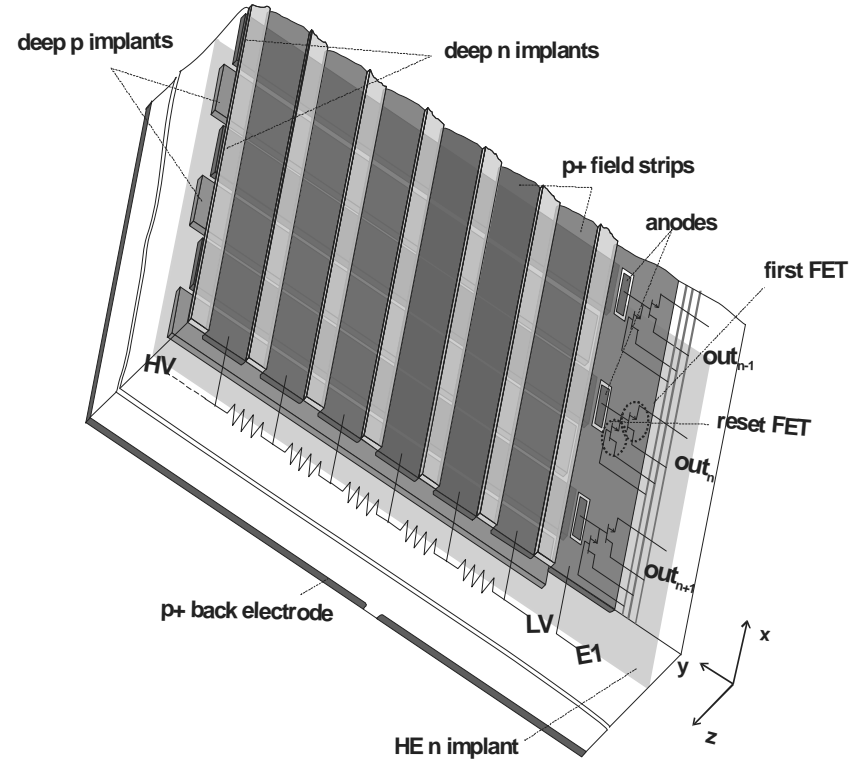

Fig. 1. Schematic 3D-view of a MLSDD with High-Energy (HE) implant to locate the potential minimum for the electrons close to the anode side of the detector.

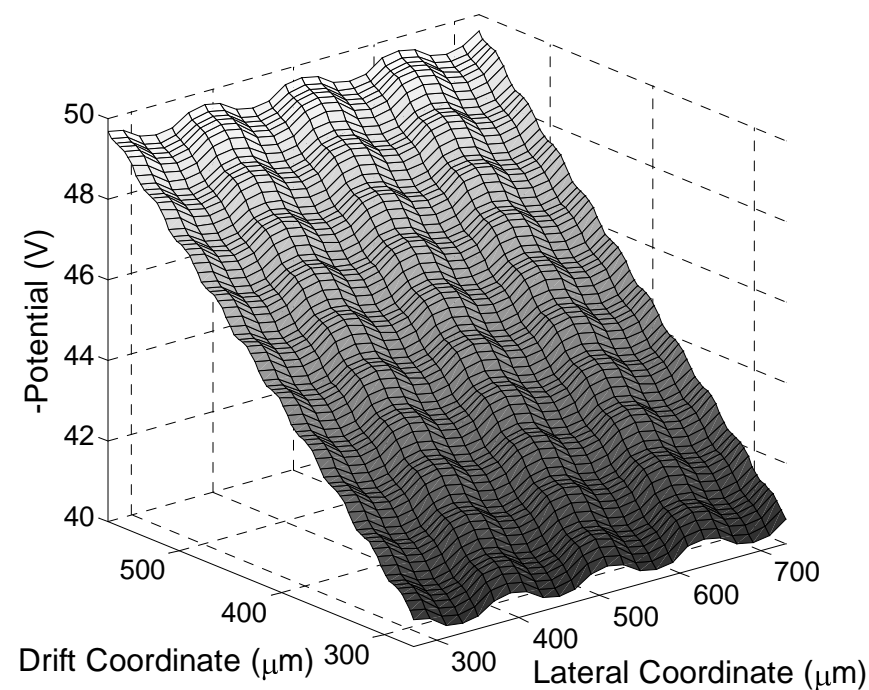

Fig. 2. 3D simulation of the electrostatic potential in a plane parallel to the MLSDD surface at the depth where the potential minimum for the electrons is located.

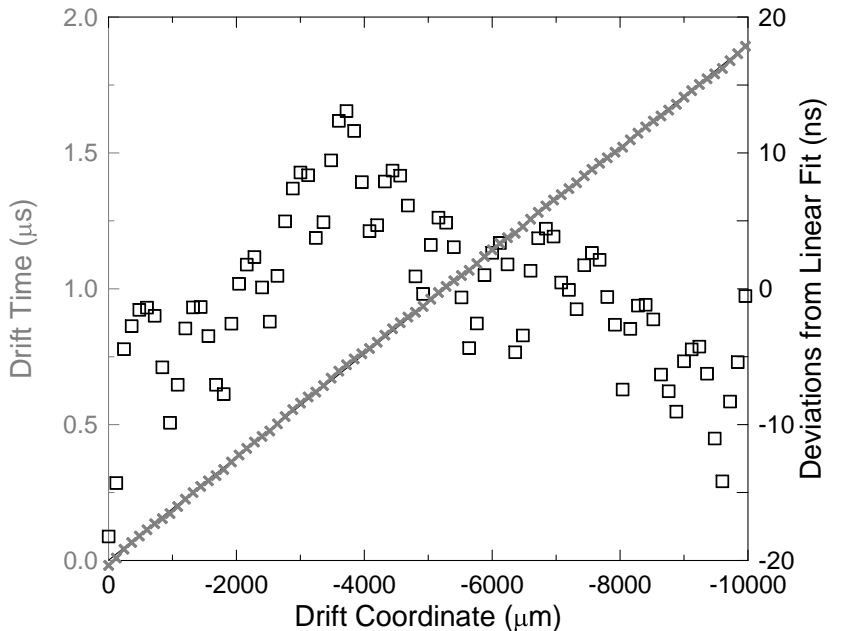

Fig. 3. Drift time as a function of the drift coordinate (gray crosses) with the detector biased at $450 \mathrm{~V} / \mathrm{cm}$ drift field measurement at $450 \mathrm{~V} / \mathrm{cm}$ drift field. The deviations from the linear fit are also shown (black empty squares).

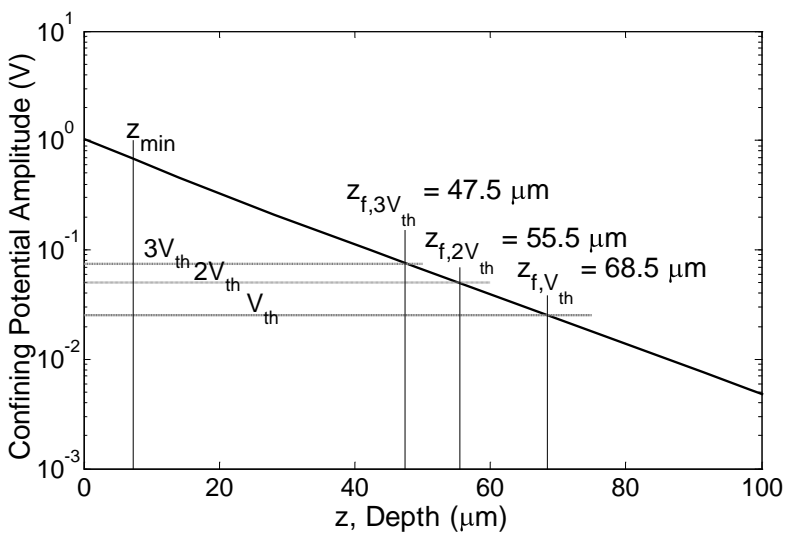

(a)

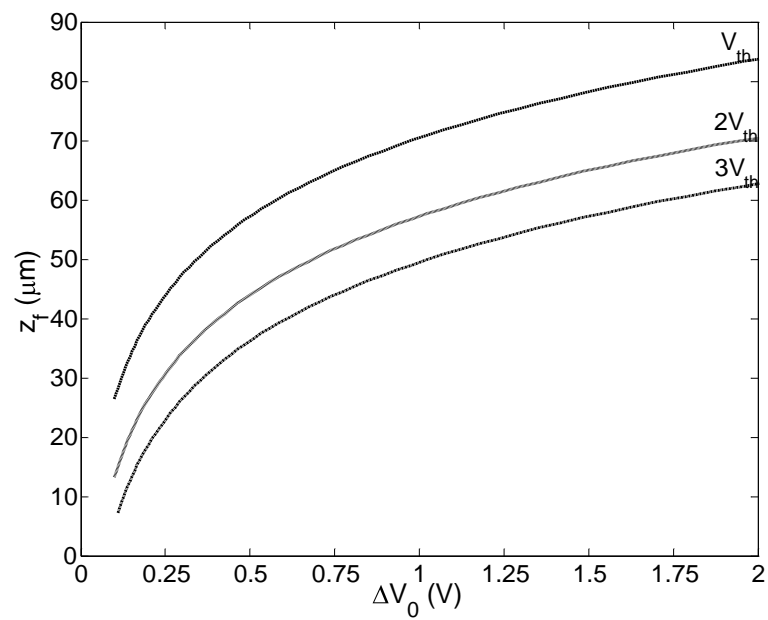

(b)

Fig. 4. a) Amplitude of the confining lateral barrier as a function of the depth according to a 1-D model. The location of the potential minimum for the electrons together with the depth at which the confining barrier equals 1,2 or

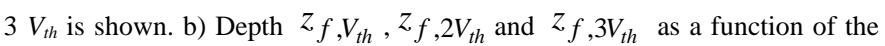
amplitude of the first harmonic of Fourier decomposition of the confining potential at the surface. 


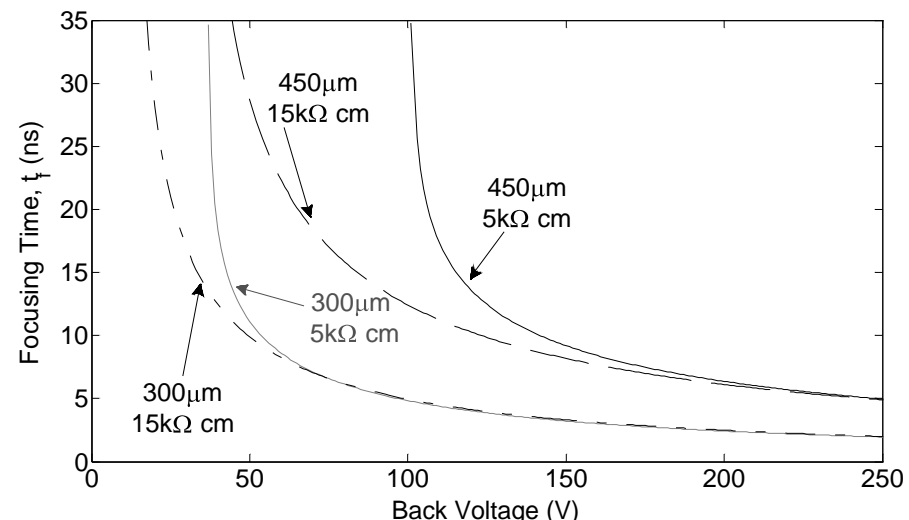

Fig. 5. Focusing time computed with (4) for two different thicknesses of the substrate (typical values of $300 \mu \mathrm{m}$ and $450 \mu \mathrm{m}$ have been chosen) and for two different dopings of the substrate $(5 \mathrm{k} \Omega \mathrm{cm}$ and $15 \mathrm{k} \Omega \mathrm{cm})$ as a function of the voltage applied at the back side, when the front side is biased at $12 \mathrm{~V}$.

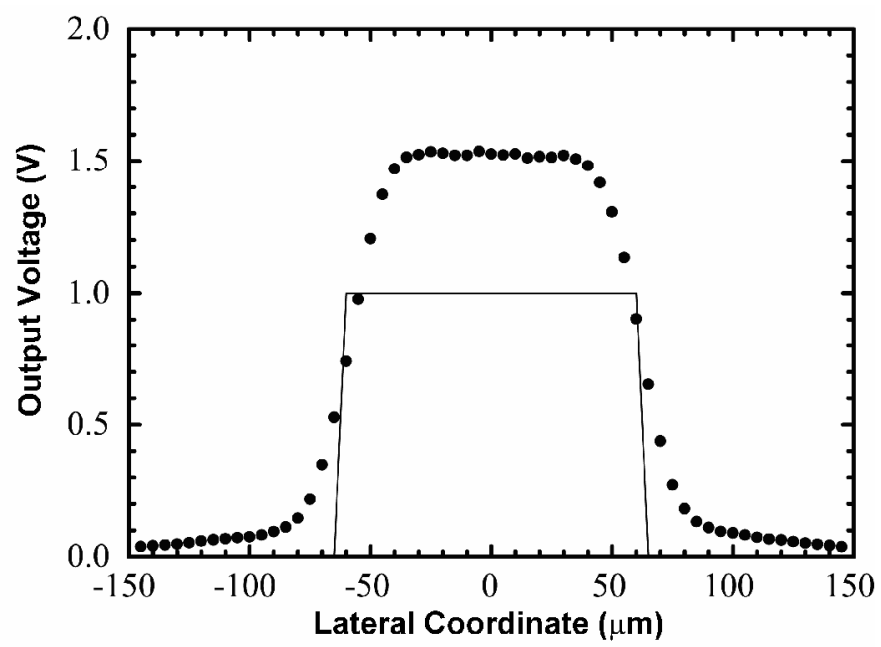

(a)

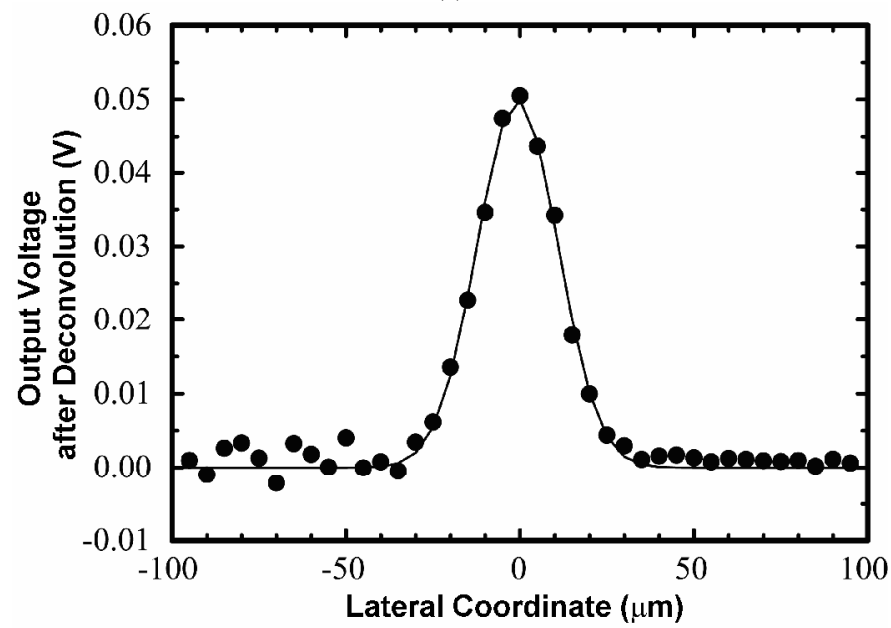

(b)

Fig. 6. Summary of the proposed fit algorithm: (a) measured charge without offset (rounds) and the weight function (solid line); (b) output voltage after deconvolution (rounds) and gaussian fit (solid line).

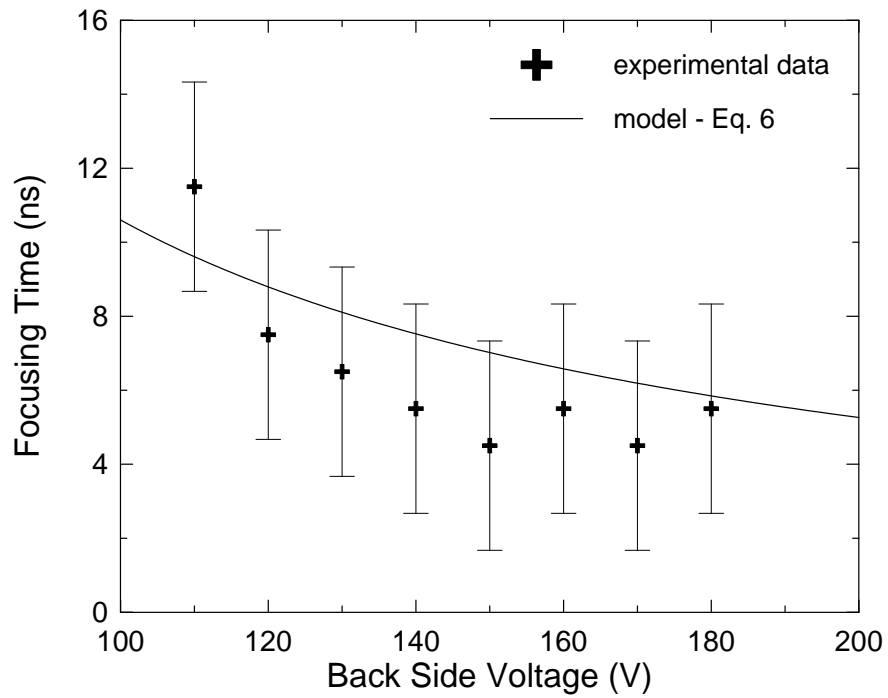

Fig. 7. Focusing time as a function of the back side voltage. The line shows the behavior foreseen by (6) for a $420 \mu \mathrm{m}$ thick $15 \mathrm{k} \Omega \cdot \mathrm{cm}$ wafer.

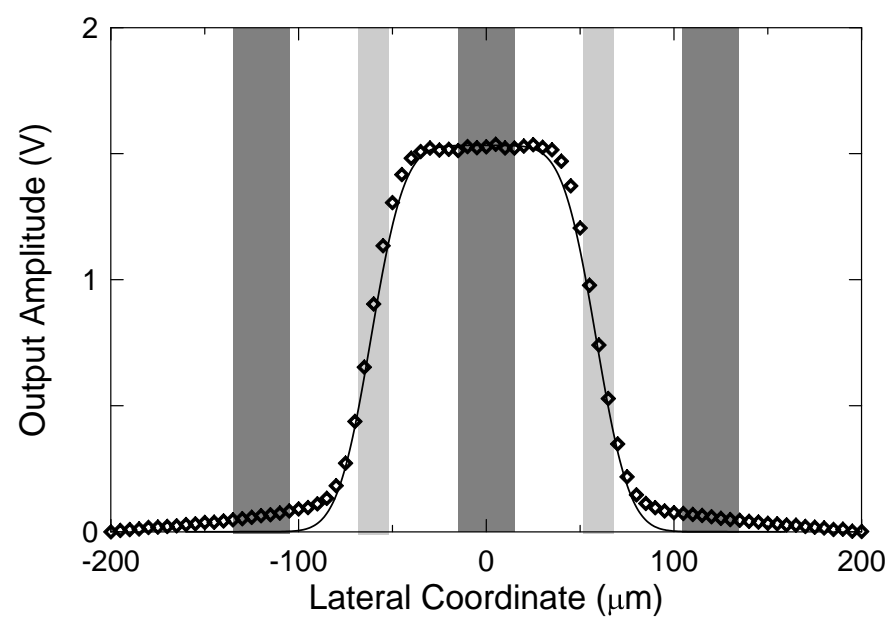

Fig. 8. Measurement of the charge collected at the anode as a function of the lateral position of the laser spot in the case of a back voltage equal to $180 \mathrm{~V}$ and of an injected charge of 6,000 electrons. The measurement is relative to a $500 \mu \mathrm{m}$ drift coordinate. The offset due to the laser spot halo has been subtracted from the measured data. The dark-gray filled areas indicate the deep $\mathrm{n}$ implants while the light-gray filled areas indicate the deep $\mathrm{p}$ implants. The solid line is the result of the least square fitting procedure.

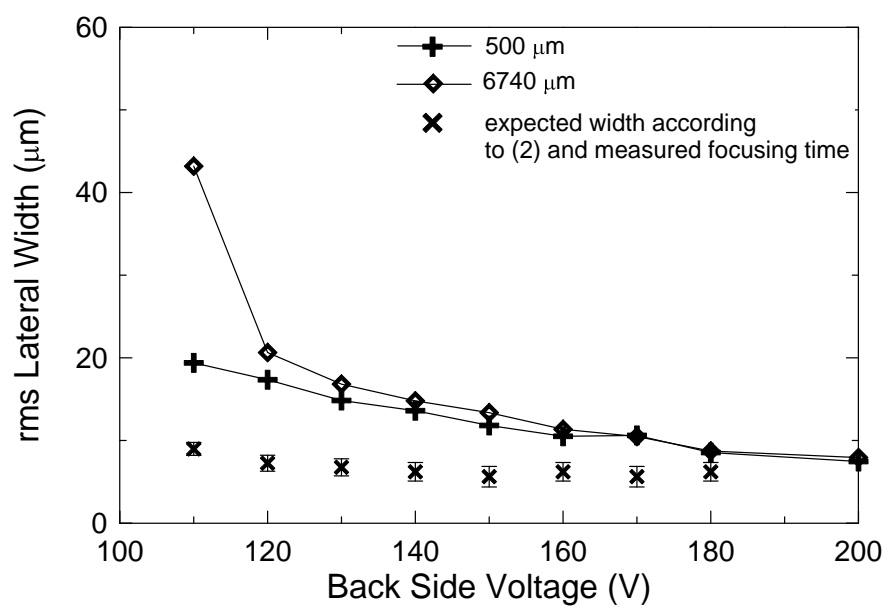

Fig. 9. Charge cloud rms lateral width as a function of the back side voltage in case of $6 \cdot 10^{3}$ injected electrons for two different drift coordinates after quadratic subtraction of the laser spot rms width. The lateral spread derived from the focusing time according to (2) is also shown. 


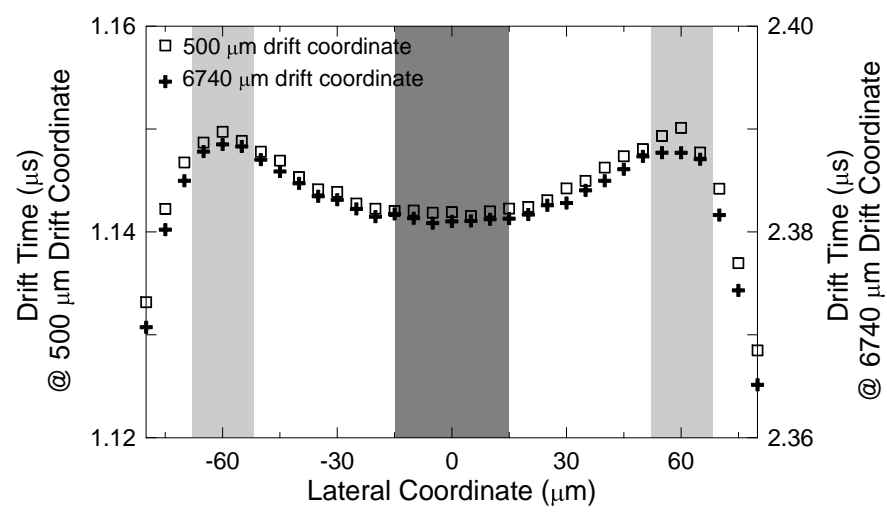

Fig. 10. Drift time as a function of the lateral position of the laser spot in the case of a back voltage equal to $180 \mathrm{~V}$ and of an injected charge of 6,000 electrons for two different drift coordinates. The dark-gray filled areas indicate the deep $\mathrm{n}$ implants while the light-gray filled areas indicate the deep p implants.

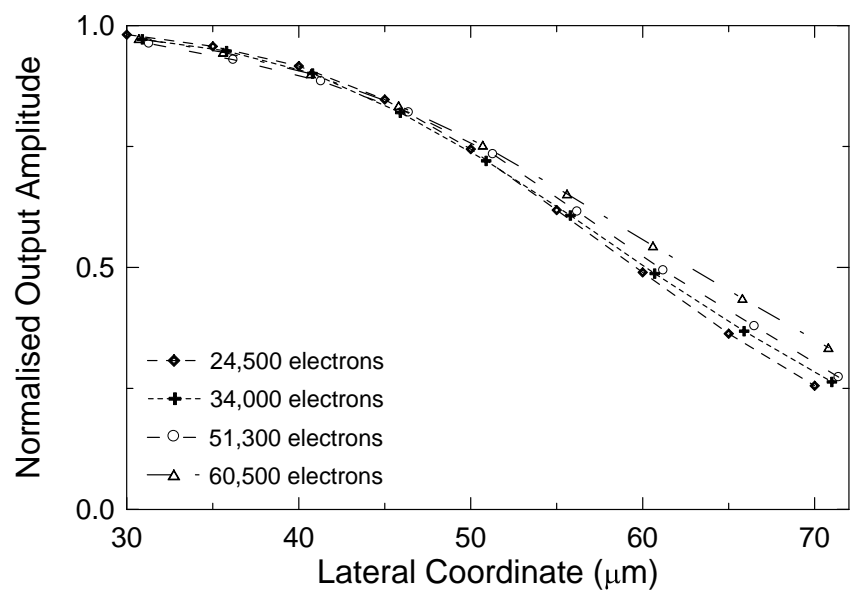

Fig. 11. Zoom of the measured collected lateral charge profile (i.e. charge collected at the anode as a function of the lateral position of the laser spot) in the case of a back voltage equal to $200 \mathrm{~V}$ for different levels of charge injection. The measurement is relative to a $6740 \mu \mathrm{m}$ drift coordinate.

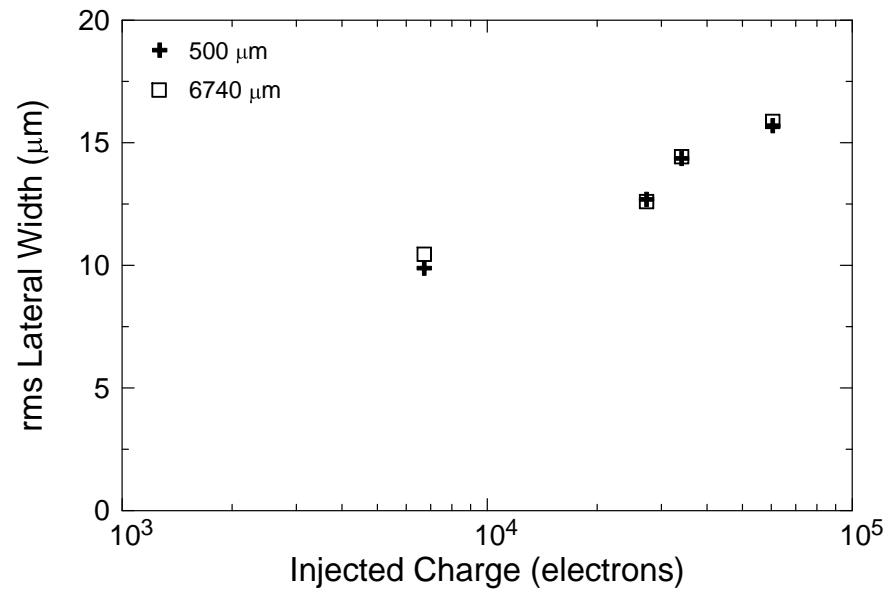

Fig. 12. Charge cloud rms lateral width (after quadratic subtraction of the laser spot rms width) as a function of the injected charge for a back side voltage of $180 \mathrm{~V}$ for two different drift coordinates.

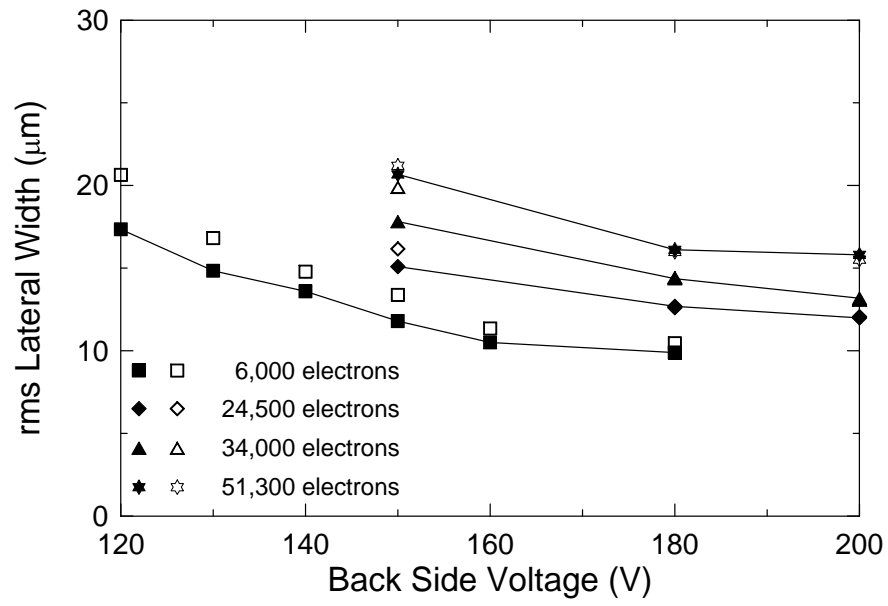

Fig. 13. Charge cloud rms lateral width (after quadratic subtraction of the laser spot rms width) as a function of the back side voltage for different levels of charge injection at $x=500 \mu \mathrm{m}$ (bold symbols) and at $x=6740 \mu \mathrm{m}$ (light symbols).

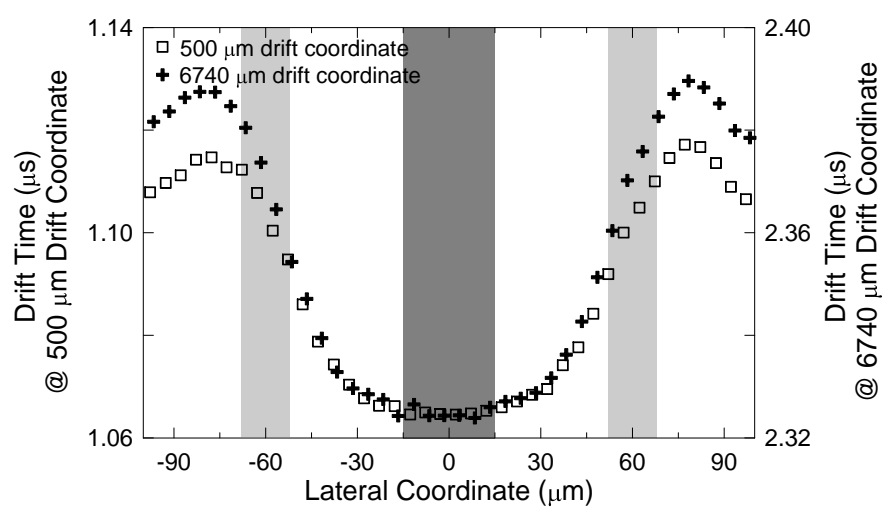

Fig. 14. Drift time as a function of the lateral position of the laser spot in the case of a back voltage equal to $200 \mathrm{~V}$ and of an injected charge of 60,000 electrons at two different drift coordinates. The dark-gray filled areas indicate the deep $\mathrm{n}$ implants while the light-gray filled areas indicate the deep $\mathrm{p}$ implants. 